\title{
Effect of post type on the fracture resistance of endodontically treated premolars
}

\author{
Ekaterina Karteva ${ }^{1,{ }^{*}}$, Neshka Manchorova ${ }^{1}$, Dessislava Pashkouleva $^{2}$, Donka Keskinova ${ }^{3}$, \\ and Stoyan Vladimirov ${ }^{1}$ \\ ${ }^{1}$ Department of Operative Dentistry and Endodontics, Faculty of Dental Medicine, Medical \\ University - Plovdiv, 4002 Plovdiv, Bulgaria \\ ${ }^{2}$ Institute of Mechanics, Bulgarian Academy of Sciences, 1113 Sofia, Bulgaria \\ ${ }^{3}$ Department of Applied and Institutional Sociology, University of Plovdiv "Paisii Hilendarski", 4000 \\ Plovdiv, Bulgaria
}

\begin{abstract}
Endodontically treated teeth (ETT) are more susceptible to fracture during mastication. The aim of our study was to investigate the fracture resistance of premolars, restored with different techniques. Eighteen human premolars, extracted for orthodontic or periodontal reasons, were used. Mesio-occlusal cavities were prepared on all of them. They were divided into 3 groups according to the restoration method: with a cemented fiber post (FP), with a cemented metal post (MP) and without a post, but restored with a fiber reinforced composite core (FRC). After thermocycling, their fracture resistance was evaluated in a standard testing machine. The results were analyzed with one-way ANOVA and Tukey Honestly Significant Difference (HSD) tests $(p<0.05)$. The fracture modes were also determined. The MP group showed the lowest fracture resistance rates and the highest number of unrestorable root fractures. In conclusion, teeth restored with FRC cores or FP show better survival rates than those restored with metal posts.
\end{abstract}

\section{Introduction}

Endodontic treatment is one of the most demanding dental procedures. Each tooth can present various challenges to the clinician, such as severe pain, curved canals and difficult access because of transposition [1]. In addition, endodontically treated teeth (ETT) are more susceptible to fracture during mastication [2]. They are reported to have shorter survival rates than vital teeth. One of the main reasons for this phenomenon is the reduction in tooth structure, which is a result of caries, trauma and cavity preparations. Other factors that influence the survival rate of these teeth are abrasion, erosion, noncarious lesions and the age of the patient [3]. Nevertheless, the primary reasons for the occurrence of partial or complete cusp fractures are the excessive loss of hard dental tissues and endodontic therapy. In many cases, the failure of the restorations results in vertical root fracture that cannot be restored, leading to the extraction of the tooth.

\footnotetext{
* Corresponding author: katya.kk@gmail.com
} 
In cases with substantial hard tissue loss, the retention of the restorative material is usually provided by a post [4]. According to the method of fabrication, there are two main groups of posts available: cast post and core systems, and prefabricated posts, upon which a direct core is placed. The materials used for the fabrication of a post also vary widely. Initially, they were made of stainless steel or precious gold alloys. Later, posts made of titanium alloys were introduced. Due to the recent developments in the field of dental materials and the advances in adhesive dentistry, posts made of fiber-reinforced resin composite or ceramic have become widely popular. In particular, the zirconium dioxide and glass fiber-reinforced posts offer a reliable bonding to the hard dental tissues, as well as a highly aesthetic result [5].

There are a large number of studies on the restoration of severely with various post systems $[6,7]$. However, the influence of post type on the fracture resistance of teeth with minor hard dental tissue loss is not extensively studied [8].

In recent years, the use of new type of core material - fiber-reinforced composite (FRC), has been widely advocated. Compared to the commercial particulate filler composite resin (PFC), it has superior mechanical properties, combined with significantly lower polymerization shrinkage [9].

With respect to the type of teeth most susceptible to fractures, the premolars show the highest failure rates [10]. This motivated us to investigate the fracture resistance of premolars with mesio-occlusal (MO) cavities, restored with either metal posts, fiber posts or FRC only. The hypotheses were:

1. Premolars with MO cavities, restored with prefabricated fiber posts and a direct composite, have a higher fracture resistance than premolars restored with metal posts;

2. Premolars, restored with prefabricated metal posts show more severe fracture modes than premolars restored with prefabricated fiber posts.

3. Premolars with MO cavities, restored with no post, but strengthened with a FRC core and a direct composite, have higher fracture resistance than premolars, restored with prefabricated posts.

\section{Materials and methods}

Eighteen human, single root premolars, free of cracks and defects were extracted for orthodontic or periodontal reasons. They were stored in $0.2 \%$ thymol solution for no longer than three months. The bucco-lingual and mesio-distal widths of the crowns were measured and only teeth of similar sizes were selected for the experiment. They were divided in 3 groups $(n=6)$ : teeth restored with a fiber post $(\mathrm{FP})$, teeth restored with a metal post (MP) and teeth restored with a FRC core and no post. MO cavities were prepared on all of the samples.

The teeth were then thermocycled for 5000 cycles between $5 \pm 5^{\circ} \mathrm{C}$ and $55 \pm 5^{\circ} \mathrm{C}$ (LTC 100, LAM Technologies, Italy). Subsequently, their roots were embedded in selfcuring resin to a level $2 \mathrm{~mm}$ apical to the cementoenamel junction (CEJ) using a modified technique, proposed by Soares et al. [11]. The periodontal ligament was simulated using a polyether-based impression material (Impregum Garant L Duo Soft, $3 \mathrm{M}$ ESPE). First, the roots were dipped into melted wax to a point $2 \mathrm{~mm}$ apical to the CEJ. Then, a radiographic film with a circular hole was used to stabilize the tooth for the embedding step. The tooth was thus positioned in a plastic cylinder $(25 \mathrm{~mm}$ in diameter and $35 \mathrm{~mm}$ in height) and the self-curing resin was inserted. After the polymerization of the resin, the teeth were removed and the wax was cleaned from the roots. The polyether was placed in the resin and the teeth were re-inserted until the setting of the material. 
The specimens were then subjected to the static fracture resistance test by using a universal testing machine (Fu1000e, Germany). They were loaded in compression at a constant speed of $4 \mathrm{~mm} / \mathrm{min}$ along the tooth axis until failure (Fig. 1). The mechanical testing is performed at a room temperature. A computer recorded the data on the forces and the displacements. The digitally recorded values are used to draw the load-strain curves and to define the fracture resistances. The results were analyzed with One-way Analysis of Variance (ANOVA). Differences between groups were identified with Tukey Honestly Significant Difference (HSD) tests. A significance level of 0.05 was used for all the tests.

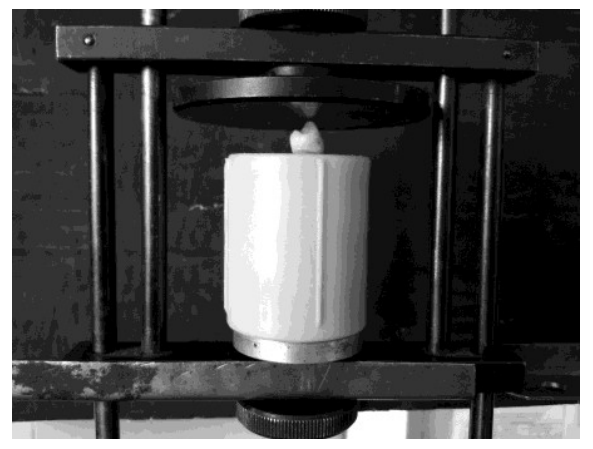

Fig. 1. Specimen in a testing machine.

The fractured specimens were visually analyzed and divided into 4 groups according to the modes of fracture. A modified classification system based on that used by Burke et al. [12] was used: 1. Fractures involving a small part of the coronal hard dental tissues (HDT); 2. Fractures involving a small part of the HDT and a cohesive fracture of the obturation; 3. Fractures involving the HDT, cohesive and/or adhesive fracture of the obturation with root involvement (that can be restored). 4. Severe fractures of the crown and root that cannot be restored (Fig. 2).

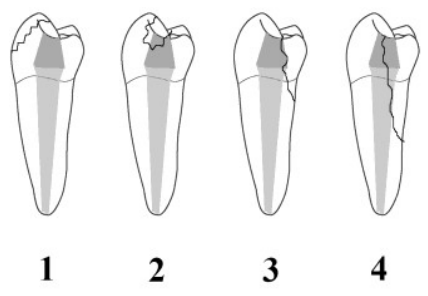

Fig. 2. Classification of the modes of fracture.

\section{Results}

Obtained typical diagrams of the load of premolars are showed in Fig. 3. The mean fracture resistance and standard deviation are illustrated in Table 1.The one-way ANOVA showed that there were significant differences among the three groups with respect to their resistance to fracture $(p<0.05)$ (Table 1$)$. The FRC group presented higher fracture resistance values, compared to the other groups. The fracture resistance between the FP and MP groups was also significantly different, with the MP showing the lowest values. 


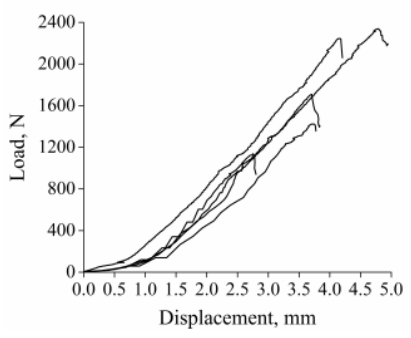

a

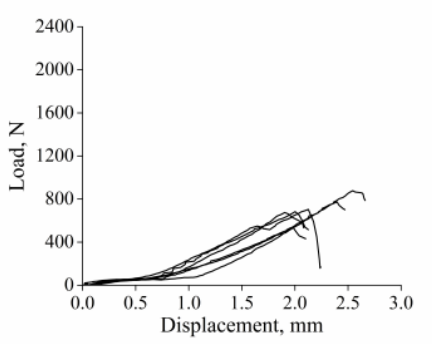

$\mathrm{b}$

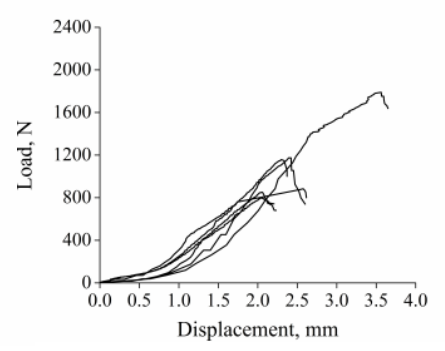

$\mathrm{c}$

Fig. 3. Load-strain diagrams of the groups: FRC (a), MP (b) and FP (c).

Table 1. Mean fracture resistance of ETT.

\begin{tabular}{|c|c|c|c|}
\hline Group & Teeth number $n$ & Fracture resistance $[\mathrm{N}]$ & Standard deviation \\
\hline FRC & 6 & 1770.5 & 517.8 \\
\hline MP & 6 & 707.6 & 113.9 \\
\hline FP & 6 & 1108.9 & 369.6 \\
\hline \multicolumn{3}{|c}{$p$ values $<0.05$} \\
\hline
\end{tabular}

Fracture mode distribution according to Fig. 2 is presented in Table 2. The MP group presented the highest incidence of fracture types 4 . The teeth in the FRC and FP group showed more favourable fracture modes.

Table 2. Fracture mode distribution among groups.

\begin{tabular}{|c|c|c|c|c|}
\hline Group & \multicolumn{4}{|c|}{ Fracture type } \\
\hline \multirow{2}{*}{ FRC } & $\begin{array}{c}\text { No of teeth, } \\
\text { assigned to } \\
\text { group 1 }\end{array}$ & $\begin{array}{c}\text { No of teeth, } \\
\text { assigned to } \\
\text { group 2 }\end{array}$ & $\begin{array}{c}\text { No of teeth, } \\
\text { assigned to } \\
\text { group 3 }\end{array}$ & $\begin{array}{c}\text { No of teeth, } \\
\text { assigned to } \\
\text { group 4 }\end{array}$ \\
\hline MP & - & 1 & 5 & - \\
\hline FP & - & - & 1 & 5 \\
\hline
\end{tabular}

\section{Discussion}

The results confirmed the first hypothesis - that teeth, restored with fiber posts show greater fracture resistance than teeth restored with metal posts. A possible reason for this is the difference in the mechanical properties of the two materials, namely - their modulus of elasticity. The mean modulus of elasticity of dentin is between 16.5 and $18.6 \mathrm{GPa}$ [13], while the values for metal and fiber posts are 210 and 39, respectively $[14,15]$. The much lower modulus of elasticity of fiber posts means that they should cause less stress to the root dentin than metal ones. Thus, by lowering the stress that occurs during mastication, the risk of root fracture should be reduced. De Castro et al. examined the influence of the post material (stainless steel, titanium or carbon/Bis-GMA posts) and post configuration on the stress distribution in ETT [16]. The results showed that the shape of the post had a 
relatively small effect on stress concentration. However, the stainless steel posts showed the highest levels of stress, compared to the other post types.

The hypothesis that premolars restored with metal posts would show more severe fractures than those restored with fiber posts was also confirmed. Our results showed the highest number of unrestorable fractures in teeth from the MP group. Compared to the other groups, they also presented the lowest fractures resistance modes. This is in agreement with the results from previous studies [17]. However, there is scientific evidence that the post material used does not significantly influence the survival rates of ETT. In a clinical longterm pilot trial, Sterzenbach et al. tested the biommetic concept that posts with low modulus of elasticity are more successful in restoring non-vital teeth [18]. The results for both materials used (fiber posts and titanium posts) were favourable after 7 years, with similar failure rates. The reasons behind this contradictory data could be several: the Young's modulus of titanium posts is $103 \mathrm{GPa}$, which is two times lower than that of the stainless steel posts used in our study [19]; all of the teeth in the clinical trial were restored with metal-fused ceramic crowns, which increased their fracture resistance. Therefore, the materials and techniques used for determining the fracture resistance of ETT vary considerably and should be taken into account when comparing the results.

The third hypothesis was also confirmed - the teeth restored with FRC used for core build-up with no post, showed higher fracture resistance compared to the other groups. FRC resin shows better flexural strength due to the introduction of short, variously orientated fibers [20]. This ensures that the toughness of the material remains the same, irrespective of the direction of the masticatory forces. The presence of the fibers also contributes towards the reduction of the polymerization shrinkage of the material. This in turn decreases the formation of micro-gaps, which are responsible for stress concentration within the restoration, micro-leakage and the formation of cracks.

\section{Conclusion}

The results of our study suggested that teeth restored with either a fiber-reinforced composite core or fiber post show better survival rates that teeth restored with stainless steel posts. Premolars, restored with no post but strengthened with a fiber-reinforced composite core have highest fracture resistance because it reinforces the material by filling the cracks. Further studies are needed to determine whether and how the extent of tissue loss can affect these results.

\section{References}

1. G. Yordanova, M. Dinkova, Compt. Rend. Acad. Bulg. Sci. 68, 107-114 (2015)

2. D. Assif, C. Gorfil, J. Prosthet Dent. 71, 565-567 (1994)

3. S.C. Khera, Z. Askarieh, J. Jakobsen, Dent. Mater., 6, 5-9 (1990).

4. I. Peroz, F. Blankenstein, K.P. Lange, M. Naumann, Quintessence Int., 36, 737-746 (2005)

5. A. Almaroof, et al, J. Dent. 43, 1308-1315 (2015)

6. M. Naumann, A. Preuss, R. Frankenberger, Dent. Mater. 23, 138-144 (2007)

7. G. Heydecke, F. Butz, A. Hussein, J.R. Strub, J. Prosthet Dent. 87, 438-445 (2002)

8. G. Heydecke, F. Butz, J.R.Strub, J. Dent. 29, 427-433 (2001)

9. S. Garoushi, P. K. Vallittu, D. C. Watts, L. V. J. Lassila, Dent. Mater. 24, 211-215 (2008)

10. A. Tamse, I. Zilburg, J. Halpern, Int. Endod. J. 31, 127-132 (1998)

11. P. V. Soares, et al., J. Prosthet. Dent. 99, 30-37 (2008) 
12. F. J. Burke, J. Dent. 20, 131-139 (1992)

13. R. G. Craig, F. A. Peyton, J. Dent. Res. 52, 710-718 (1958)

14. A. Lanza, R. Aversa, S. Rengo, D. Apicella, A. Apicella, Dent. Mater. 21, 709-715 (2005)

15. T. Nakamura, et al., Dent. Mater. J. 24, 275-279 (2005)

16. R. de Castro Albuquerque, et al., J. Oral Rehab. 30, 936-943 (2003)

17. C. J. Goodacre, J. Evid. Based Dent. Pract. 10, 32-34 (2010)

18. G. Sterzenbach, Al. Franke, M. Naumann, J. Endod. 38, 1557-1563 (2012)

19. C. S. M. Coelho, et al., Dent. Mater. J. 28, 671-678 (2009)

20. S. Garoushi, P. K. Vallittu, L. V. J. Lassila, Dent. Mater. 23, 1356-1362 (2007) 